\title{
Complications of Percutaneous Endoscopic Lumbar Discectomy: Experiences and Literature Review
}

\author{
Bin Zhu ${ }^{1}$, Yi Jiang ${ }^{2}$, Lanpu Shang ${ }^{1}$, Ming Yan ${ }^{3}$, Hai-Jun Ma ${ }^{4}$, Da-Jiang Ren ${ }^{5}$ and Xiao-Guang Liu ${ }^{1^{*}}$ \\ ${ }^{1}$ Department of Orthopaedics, Pain Management Center, Peking University Third Hospital, Beijing, PR China \\ ${ }^{2}$ Department of Orthopaedics, Beijing Haidian Section, Peking University Third Hospital, Beijing, PR China \\ ${ }^{3}$ Department of Spine, The First Hospital of Jilin University, Changchun, PR China \\ ${ }^{4}$ Disc Disease Center, Third People's Hospital of Henan Province, Zhengzhou, PR China \\ ${ }^{5}$ Department of Orthopaedics, People's Liberation Army General Hospital, Beijing, PR China
}

*Corresponding author: Xiao-Guang Liu, Department of Orthopaedics, Pain Management Center, Peking University Third Hospital, Beijing, PR China, Tel: +8613488737864; E-mail: zhubin@bjmu.edu.cn

Rec Date: December 06, 2017; Acc Date: December 18, 2017; Pub Date: December 23, 2017

Copyright: $\odot 2017$ Zhu B, et al. This is an open-access article distributed under the terms of the Creative Commons Attribution License, which permits unrestricted use, distribution, and reproduction in any medium, provided the original author and source are credited.

\begin{abstract}
Study design: A retrospective clinical review.

Purpose: To explore the type, morbidity, risk factors and treatment strategies of postoperative complication following percutaneous endoscopic lumbar discectomy (PELD) surgery.

Overview of literature: PELD became one of the main operation methods for degenerative lumbar diseases, including disc herniation, stenosis and discogenic low back pain. However, complications following PELD surgery are a considerable challenge for spinal surgeons and seldom addressed publicly.

Methods: 10120 patients after PELD surgery were studied. These surgeries were finished by 6 surgeons from 3 main minimal invasive spine centers from January 2012 to June 2017. Most of patients are regularly followed up to explore the type, morbidity, risk factors and treatment strategies of postoperative complication following PELD surgery.

Results: There are 2 patients died in the perioperative period and 2 patients with permanent impairment of neural function after surgery, which should be the severest complication of PELD surgery. Transient paresthesia, intraoperative bleeding and dura sac tear are the most common complications reported by 6 surgeons. There are 2 suspected cases of postoperative hematoma, several cases of surgical instruments broken during the surgery and 20 cases of infection in 10120 patients, regarded as rare complications of PELD. Recurrence rate of PELD surgery is $4.7 \%$ to $6 \%$ reported by 3 surgeons. However, recurrence defined as complications of PELD surgery remain controversial.
\end{abstract}

Conclusion: Excellent clinical outcome of large case series after PELD surgery is reported. However, we need to face the limitations and complications of the surgery. The complication rate should be reduced by caring about the treatment, surgical indications strictly selected and the guidance of experienced surgeons.

Keywords: Discectomy; Percutaneous; Endoscopy; Postoperative complications; Risk factors; Retrospective studies

\section{Introduction}

Since Yeung introduced the rigid rod-lens, integrated, multichannel, wide-angle operating spinal endoscope system (Yeung Endoscopic Spinal System, YESS) since 1991, percutaneous endoscopic lumbar discectomy (PELD) became one of the main operation methods for degenerative lumbar diseases, including disc herniation, stenosis and discogenic low back pain [1-9]. Different tools, techniques and skills were invented, and the indications of endoscopic spinal surgery expanded from lumbar diseases to cervical and thoracic spine, spinal infection and spinal neoplasms. Thousands of papers on PELD have been published; however, few authors discussed the complications and treatment strategies.

\section{Materials and Methods}

We interviewed 6 surgeons from 3 main minimal invasive spine centers (including our center). 10120 cases of PELD surgeries were finished in these 3 centers from January 2012 to June 2017. Each surgeon performed more than 1000 cases of PELD surgeries.

There are 4 main questions: 1) What are the severest complications of PELD you met or heard? 2) What are the most common complications of PELD you met or heard? 3) What are the rarest complications of PELD you met or heard? 4)Treatment strategy and clinical outcome. 


\section{Results}

\section{The severest complications of PELD}

Perioperative death may be the severest complication of PELD surgery. We heard but not met 2 patients died in the perioperative period. The first patient felt sudden chest pain 6 hours after the PELD surgery and was diagnosed as acute heart infarction. He died 18 hours after the surgery. The correlation between heart infarction and PELD surgery was unknown. The second patient died of rupture of abdominal aorta during the placement of working tube. The wrong direction of puncture and "foraminoplasty", short of experience were thought to be the main risk factors. Figure 1 showed the wrong direction of puncture.

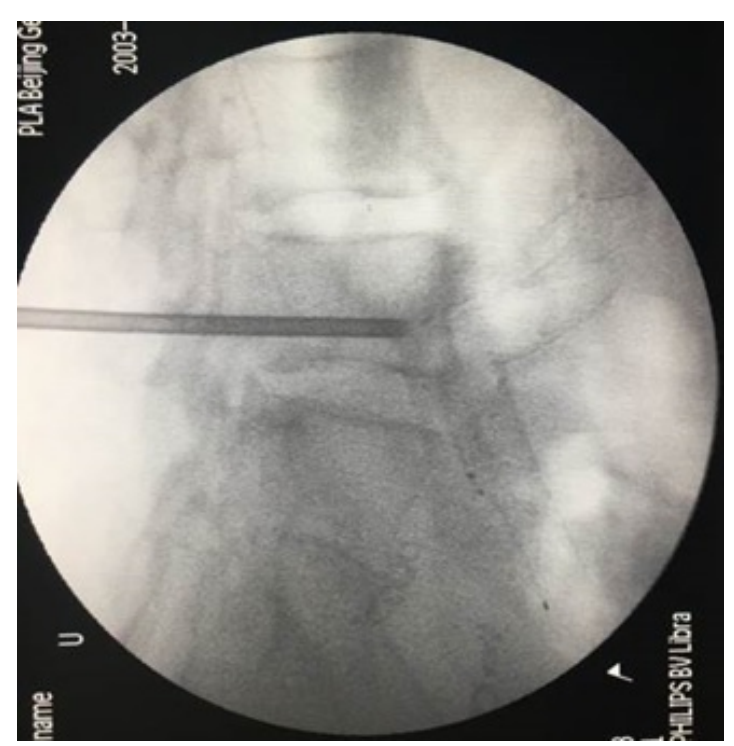

Figure 1: The wrong direction of puncture needle increased the risk of injury of lumbosacral plexus, blood vessels and abdominal viscera.

Two surgeons met permanent impairment of neural function and thought it to be the severest complication of PELD too. The first patient was female. She suffered radicular pain due to disc herniation of L4-5, dura sac was teared intraoperative (Figure 2) and the patient felt severe head and neck pain, decompression was sufficient, but the muscle strength grade of tibialis anterior and hallux extensor was 0 after surgery. The nerve function deficit remained unchanged after 3 years' follow-up. The reasons were thought to be dura sac tear and high-water pressure. The second patient was male, he also suffered radicular pain due to disc herniation of L4-5, no signs of nerve injury or complain of radicular pain were noted during the operation. The muscle strength grade of quadriceps femoris was 1, his function of knee extension recovered 1 year after surgery under conservative treatment but the vastus medialis atrophied at last follow-up. Compression of working tube to the exiting nerve root was suspect to pathogenesis.

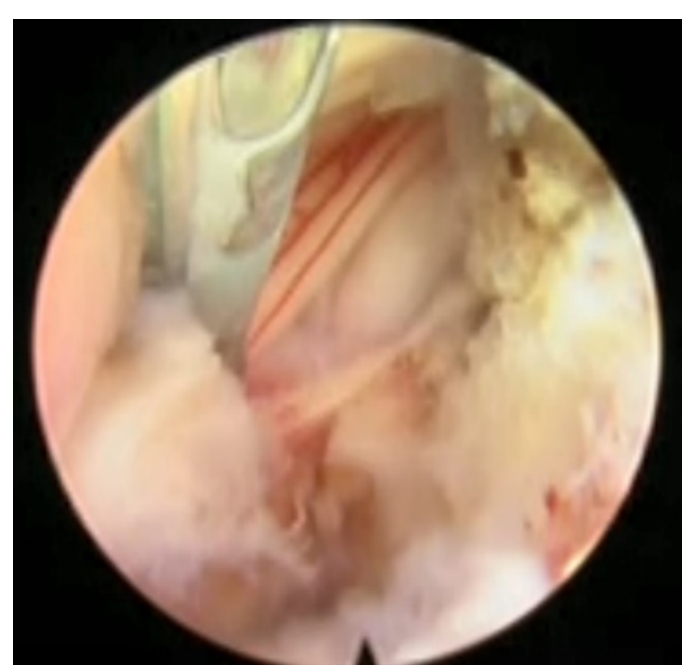

Figure 2: Teared dura sac was recorded but the surgeon didn't cut off the nerve root.

\section{The most common complications}

Six surgeons reports various common complications of PELD surgeriesincluding transient paresthesia, intraoperative bleeding/ postoperative hematoma and dura sac tear.

\section{Transient paresthesia}

The estimated incidence rate reported by the 6 surgeons was $4.0 \%$ (406/10120). Most cases of transient paresthesia were caused by the compression of working tube to the exiting nerve root. Risk factors included reduced height of the foramen, intraforaminal or far lateral disc herniation and wrong position of the working tube. Most patients recovered after several weeks or months without additional therapeutic measures.

\section{Intraoperative bleeding/postoperative hematoma}

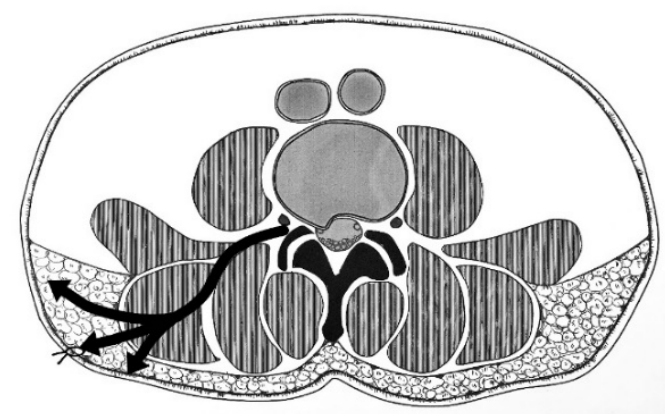

Figure 3: The blood could permeate through the split of lumbodorsal fascia to the subcutaneous soft tissue. 
Intraoperative bleeding was common and annoyed, which occurred in 153 cases. Injury of internal vertebral venous plexus and cancellous bone were the main cause of bleeding. Bipolar radiofrequency probe and increasing perfusion pressure would take effect in most cases. However, postoperative hematoma was rarely reported, only 2 surgeons reported 2 suspected cases of hematoma due to recurrent radicular pain within 12 hours after the surgery and relieved within 3 days. No additional open or minimal invasive surgery was needed. The blood could permeate through the split of lumbodorsal fascia to the subcutaneous soft tissue (Figure 3 ).

\section{Dura sac tear}

The rate of dura sac tear was much higher than the reported rate in literature. Most cases were asymptomatic. Wrong identification of endoscopic structure, adhesion and rough operation of sharp tools (trephine, scissors punch) were the main risk factors. In most cases, drainage was not necessary.

In our study, there were 482 cases with dura sac tear. There was a special case of dura sac tear in which the nerve root herniated from the split and formed a strangulated nerve root hernia (Figure 4). This condition was extremely rare, and the patient got paroxysmal radicular pain. The symptom lasted 3 months and relieved with oral analgesic. Some authors reported reoperation and repaired the dura sac microscopically $[10,11]$.

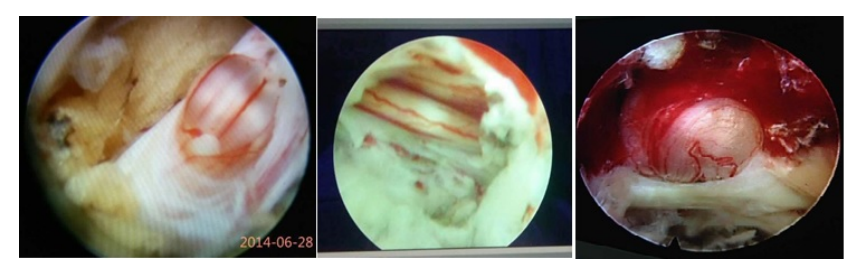

Figure 4: (A, B) Asymptomatic dura sac injury. (C) Strangulated nerve root hernia.

\section{Rare complications of PELD}

Some rare complications has been described in the previous chaptersuch as permanent impairment of neural function, strangulated nerve root hernia, postoperative hematoma. Besides, there were some other complications we met rarely but needed attention.

\section{Broken surgical instruments}

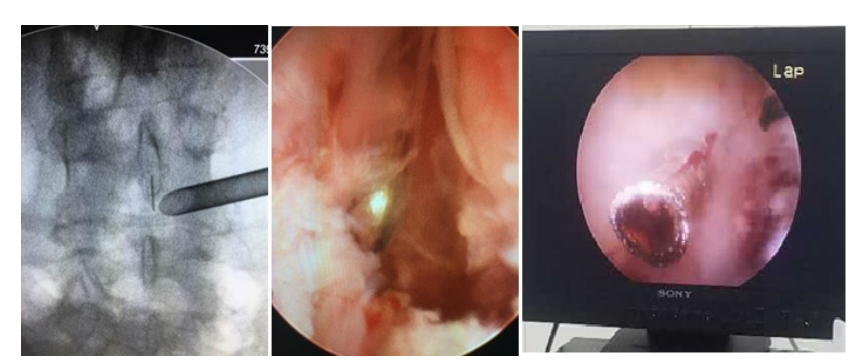

Figure 5: $(\mathrm{A}, \mathrm{B})$ Broken guide wire and hid in the disc space, it was removed by grasper. $(\mathrm{C})$ Broken diamond bur.
Metal fatigue due to repeated use and rough operation were the main risk factors. We met or heard different kinds of broken surgical instruments, such as the guide wire, biopsy forceps, diamond bur et al. (Figure 5). Checking every surgical instrument carefully before and after surgery could help avoiding such complications.

\section{Infection}

Compared with open lumbar surgery, the infection rate of PELD surgery was much lower. The estimated infection rate reported by the 6 surgeons was $0.2 \%(20 / 10120)$. Diabetes, immunosuppression, prolonged operation time, unqualified sterilization were main risk factors. Postoperative infection occurred 2-4 weeks after surgery. Back pain and immobility of lumbar spine were common symptoms. More than half cases could be cured by intravenous or oral antibiotics. Less than $1 / 3$ cases needed debridement and drainage (Figure 6).

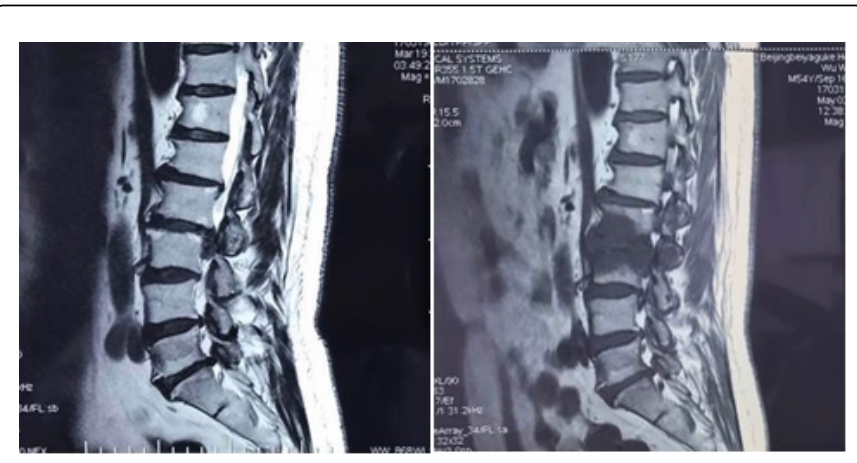

Figure 6: (A) Preoperative MRI image showed disc prolapse of L2-3. (B) The patient felt severe back pain 2 weeks after PELD surgery and MRI image showed signs of intervertebral infection, after receiving 1 week of intravenous antibiotic (third generation cephalosporin) and 2 weeks of oral antibiotic, his symptoms disappeared.

\section{Recurrence}

It remained controversial to define recurrence as complications of PELD surgery. The diagnostic criteria of recurrence was as follows [12]: 1) remission of the preoperative symptoms lasted at least 2 weeks; 2) recurrent symptoms and confirmed by MRI examination. 3 surgeons reported the recurrence rate and risk factors in the last 5 years. The detailed information was listed in Table 1.

\section{Discussion}

The numbers of percutaneous endoscopic lumbar discectomy increased rapidly in the recent 30 years in China. Zhang concluded that estimated 50,000-60,000 endoscopic spinal surgeries were performed in China every year. In 2016, nearly 200-300 Chinese doctors could perform endoscopic spine surgery with more than 100 surgeons able to successfully complete more than 100 cases/year. Hundreds of meeting and courses focus on endoscopic spinal surgeries were held in China. However, most doctors preferred to report operative skills, technic innovation and excellent clinical outcome of large case series. Few surgeons liked to discuss the complications and learning curve of endoscopic spinal surgeries. 
Page 4 of 6

\begin{tabular}{|c|c|c|c|c|c|c|}
\hline Surgeons & Recurrence rate & \multicolumn{4}{|c|}{ Risk factors of recurrence } \\
\hline Surgeon A & $4.7 \%(70 / 1540)$ & $\begin{array}{c}\text { End-plate } \\
\text { osteochondritis }\end{array}$ & $\begin{array}{c}\text { Disc degeneration } \\
\text { (Pfirmann Grade or } \\
\text { more) }\end{array}$ & Aged patients & Central herniation & Hyperactivity \\
\hline Surgeon B & $4.8 \%(52 / 1080)$ & $\begin{array}{c}\text { End-plate } \\
\text { osteochondritis }\end{array}$ & $\begin{array}{c}\text { Rupture of fibrous } \\
\text { annulus }\end{array}$ & Male(Hyperactivity) & Overweight & Dyperactivity \\
\hline Surgeon C & $6 \%(450 / 7500)$ & $\begin{array}{c}\text { End-plate } \\
\text { osteochondritis }\end{array}$ & $\begin{array}{c}\text { Decreased } \\
\text { intervertebral disc } \\
\text { height }\end{array}$ & Disc degeneration & - \\
\hline
\end{tabular}

Table 1: Recurrence of PELD surgery.

We have to know that complications following PELD surgery are a considerable challenge for spinal surgeons.

For the purpose of investigating the complications of PELD surgeries in China, we started this survey one year ago in different "WeChat" groups (popular social networking software of China). At first, the biggest problem we encountered was the "definition" of complications, surgeons reported various kinds of complications. Then we limited the survey to 6 experienced endoscopic spinal surgeons based on 10120 cases in the last 5 years.

As mentioned earlier, perioperative death and permanent impairment of neural function may be the severest complication of PELD surgery. Perioperative deaths may be caused by a variety of factors, include age, potential risks of other organs or systems. Due to surgical stress, the patient's organs and systems become dysfunctionnal and eventually cause death. It may also be associated with surgical procedures. An aortic rupture was observed in this group of patients.

The incidence of exiting root injury has been variably reported from $1.0 \%$ to $6.7 \%$ [13]. Compression of working tube to the exiting nerve root was suspect to pathogenesis. Choi et al. [13] define postoperative dysesthesia or motor weakness as a postoperative exiting root injury. They recommend measuring the distance from the exiting root to the facet at the lower disc level according to a preoperative MRI scan. If the distance is narrow, an alternative surgical method, such as microdiscectomy or conventional open discectomy, should be considered. So, we need to read MRI images carefully before surgery and select appropriate indications for surgical treatment. Transient paresthesia is a common complication of PELD surgeries reported by 6 surgeons, which is also thought to be caused by the compression of working tube to the exiting nerve root. Reduced height of the foramen is the risk factor. Yokosuka et al. [14] reported the posterolateral approach for percutaneous endoscopic lumbar discectomy can be safely used to treat foraminal and extraforaminal LDH with foraminal height $\geq 13 \mathrm{~mm}$ and foraminal width $\geq 7 \mathrm{~mm}$. We also think that the small foramen (foraminal height $13 \mathrm{~mm}$ ) increases the risk of transient paresthesia. Unlike impairment of neural function, most patients recovered after several weeks or months without additional therapeutic measures.

Intraoperative bleeding was common and annoyed. By contrast, postoperative hematoma following PELD was rare and rarely reported. Ahn et al. [15] report that a total of 4 patients (0.97\%) experienced symptomatic postoperative retroperitoneal hematoma. Inguinal pain was the common symptom in these patients. Two patients with massive hematoma compressing the intraabdominal structures required open hematoma evacuation performed by general surgeons, and the other 2 patients with small, localized hematoma of $<100 \mathrm{ml}$ were treated conservatively. An acute psoas muscle hematoma following PELD is reported by Kim et al. [16]. In this surgery, it is likely that injury of the segmental artery occurred during insertion of the endoscope. Preoperative computed tomography image shows the space of the disc in which the material herniated far laterally is closely connected with the running lumbar segmental artery. This patient suffered the development of acute flank and leg pain with hypotension after PELD, which suggest the formation of a psoas muscle hematoma. In conclusion, it is important to read the X, CT and MRI images carefully before surgery. Patient with medical problems or previous operative scarring is needed to be noticed. At the same time, skilled surgical procedures and adequate understanding of anatomy are important factors in avoiding hematoma.

The prevalence of unintended dura sac tear during lumbar disc surgery is variable, ranging from $1.8 \%$ to $17.4 \%$ [11]. Actually, because of the operation field filled with irrigation solution, it is difficult to detect a dura sac tear during the procedure. Most cases were asymptomatic. Once the nerve root herniates from the split and forms a strangulated nerve root hernia, intractable radicular pain breaks out. Reoperation to repair the dura sac is a solution. Far-Migrated Disc Herniation may be a risk factor of dura sac tear [17], but the results of PELD for patients with Far-Migrated Disc Herniation shows excellent in several surgeons' reports [18-21]. Many surgeons like to expose the neural tissues for thorough decompression. In my opinion, this is not necessary. The definitive end point of the procedure is free mobilization of neural tissues, not direct exposure of neural tissues [22]. As experience increases, the prevalence of dura sac tear will decrease [11].

The surgical instruments breakage in PELD procedures is a rare but severe complication, which requires immediate removal. 2 cases of guidewire breakage during PELD procedure was described in previous literature [23]. Finally, the operator inserted the working cannula to the broken end of the guidewire and retrieved it with straight grasping forceps. These patients recovered well after operation. Metal fatigue and an appropriate manner may be the reasons. A larger needle entry angle is always needed at L5-S1 level, where the guidewire is more likely to get broken by bone tissue, such as intervertebral joint, iliac crest and so on. If it is difficult to remove the broken instrument, an open procedure still needs to be considered. Fortunately, the broken instrument was also removed successfully under the endoscopy in our cases. Checking every surgical instrument carefully before and after surgery, step by step operation under the guidance of the $\mathrm{C}$ arm may be the preventing strategy. 
The infectious complications of PELD and its incidence have been seldom reported in previous literature. Constant water flow during surgery may be the reason why the infection is rare. The estimated infection rate reported by 6 surgeons was $0.2 \%$ (20/10120). However, the pathogenesis of infection still remains unclear. Repeated needling and the inadvertent direct puncture of the colon with too steep angle is a probable cause of infection [24]. Other risk factors include diabetes, immunosuppression, prolonged operation time, unqualified sterilization et al. Antibiotics is the most important method of treatment. A researcher believe that surgical treatment is usually necessary with the following [24]: 1) the presence of a neurological deficit; 2) increased paravertebral or epidural abscess formation; 3) significant disc space narrowing with adjacent vertebral body destruction; 4) spinal instability with developing kyphosis; and 5) failure to respond to conservative therapy. Another case series [25] shows that $12(0.12 \%)$ of the 9821 patients were identified as having postoperative infections, and in all these cases, the infection manifested as spondylodiscitis. We believe the best way to manage postoperative spondylodiscitis is prevention. Standard operation and disinfection are the most important.

Sometimes, we cannot make a distinction between recurrence and incomplete decompression. An immediate postoperative MRI may show the complete or incomplete decompression. Kim et al. [12] finished a series of 4861 patients with PELD surgery. They find out that age, presence of the Modic changes, protrusion-typed disc herniation could have affected the success or failure of the procedure. Lee et al. [26] think that open surgery may be considered for herniations with high-canal compromise and high-grade migration, and PELD can be considered to be a surgical option in the remaining intracanal disc herniations. Recurrence rate of PELD surgery is $4.7 \%-6 \%$ reported in our study. Age, hyperactivity, overweight, degeneration and type of herniation are the risk factors to increase the recurrence rate. Therefore, for successful PELD, it is desirable for the surgeons to consider the risk factors carefully.

A lot of reports recently have demonstrated that PELD learning curve seems to be stable and acceptable with proper pre-PELD training [8,27-30]. As surgeons gained familiarity with the procedure, the outcomes also improved. Wang et al. [31] reported that the failure rate during the early use of the PELD technique (Cases 1-70) was $17.1 \%$; then fell to $5.7 \%$ (Cases 141-210) before finally stabilizing at $10.0 \%$ (Cases 211-280 and Cases 281-350). In our study, reduction of operation time is also observed. We think most of complications occurred in the early stage of developing this new technology. However, some complications such as transient paresthesia intraoperative bleeding/postoperative hematoma, broken surgical instruments due to metal fatigue et al are unavoidable despite sufficient preparation and improved surgical skill. Compared with the transforaminal approach, the learning curve of the interlaminar approach was flat and hard to master [27]. The surgeon should start with simple cases first with the guidance of experienced surgeons. Kim et al. [32] reported that percutaneous endoscopic interlaminar lumbar discectomy with annular sealing may be a useful technique for reducing early recurrence.

\section{Conclusion}

In summary, age, hyperactivity, overweight, degeneration and type of herniation are the risk factors. Lack of experience, such as wrong position of the working tube, wrong identification of endoscopic structure and rough operation of sharp tools is thought to be the main risk factors for surgeons newly performing PELD surgery. We need to face the limitations and complications of the surgery. The complication rate should be reduced by caring about the treatment, surgical indications strictly selected and the guidance of experienced surgeons.

\section{Limitations}

This study has several limitations. First, it was a retrospective study. Second, there was no control group. Third, it is difficult to accurately estimate the incidence of dura sac tear. Good statistical experimental design may provide more useful information.

\section{Acknowledgement}

No funds were received in support of this work. No benefits in any form have been or will be received from a commercial party related directly or indirectly to the subject of this manuscript.

\section{References}

1. Peng CW, Yeo W, Tan SB (2009) Percutaneous endoscopic lumbar discectomy: Clinical and quality of life outcomes with a minimum 2-year follow-up. J Orthop Surg Res 4: 20.

2. Ruetten S, Komp M, Merk H, Godolias G (2007) Use of newly developed instruments and endoscopes: Full-endoscopic resection of lumbar disc herniations via the interlaminar and lateral transforaminal approach. J Neurosur Spine 6: 521-530.

3. Tzaan WC (2007) Transforaminal percutaneous endoscopic lumbar discectomy. Chang Gung Med J 30: 226-234.

4. Tenenbaum S, Arzi H, Herman A, Friedlander A, Levinkopf M (2011) Percutaneous posterolateral transforaminal endoscopic discectomy: Clinical outcome, complications, and learning curve evaluation. Surg Technol Int 21: 278 .

5. Kafadar A, Kahraman S, Akbörü M (2006) Percutaneous endoscopic transforaminal lumbar discectomy: A critical appraisal. Minim Invasive Neurosurg 49: 74-79.

6. Ruetten S, Komp M, Merk H, Georgios G (2008) Full-endoscopic interlaminar and transforaminal lumbar discectomy versus conventional microsurgical technique: a prospective, randomized, controlled study. Spine 33: 931.

7. Eun SS, Lee SH, Sabal LA (2016) Long-term follow-up results of percutaneous endoscopic lumbar discectomy. Pain Physician 19: 1161-1166.

8. Ahn SS, Kim SH, Kim DW, Lee BH (2016) Comparison of outcomes of percutaneous endoscopic lumbar discectomy and open lumbar microdiscectomy for young adults: A retrospective matched cohort study. World Neurosurg 86: 250-258.

9. Yeung AT (2007) The evolution and advancement of endoscopic foraminal surgery: One surgeon's experience incorporating adjunctive technologies. SAS Journal 1: 108-117.

10. Tamaki Y, Sakai T, Miyagi R, Nakagawa T, Shimakawa T, et al. (2015) Intradural lumbar disc herniation after percutaneous endoscopic lumbar discectomy: case report. J Neurosurg Spine 23: 336-339.

11. Ahn Y, Lee HY, Lee SH, Lee JH (2011) Dural tears in percutaneous endoscopic lumbar discectomy. Eur Spine J 20: 58-64.

12. Kim JM, Lee SH, Ahn Y, Yoon DH, Lee CD, et al. (2007) Recurrence after successful percutaneous endoscopic lumbar discectomy. Minim Invasive Neurosurg 50: 82.

13. Choi I, Ahn JO, So WS, Lee SJ, Choi IJ, et al. (2013) Exiting root injury in transforaminal endoscopic discectomy: Preoperative image considerations for safety. Eur Spine J 22: 2481-2487.

14. Yokosuka J, Oshima Y, Kaneko T, Takano Y, Inanami H, et al. (2016) Advantages and disadvantages of posterolateral approach for percutaneous endoscopic lumbar discectomy. J Spine Surg 2: 158-166. 
Citation: Zhu B, Jiang Y, Shang L, Yan M, Ma HJ, et al. (2017) Complications of Percutaneous Endoscopic Lumbar Discectomy: Experiences and Literature Review. J Spine 6: 402. doi:10.4172/2165-7939.1000402

Page 6 of 6

15. Ahn Y, Kim JU, Lee BH, Lee SH, Park JD, et al. (2009) Postoperative retroperitoneal hematoma following transforaminal percutaneous endoscopic lumbar discectomy. J Neurosurg Spine 10: 595.

16. Kim HS, Ju CI, Kim SW, Kim JG (2009) Huge Psoas Muscle hematoma due to lumbar segmental vessel injury following percutaneous endoscopic lumbar discectomy. J Kr Neurosurg Soc 45: 192.

17. Ahn Y, Lee SH, Lee JH, Kim JU, Liu WC (2009) Transforaminal percutaneous endoscopic lumbar discectomy for upper lumbar disc herniation: Clinical outcome, prognostic factors, and technical consideration. Acta Neurochirurgica 151: 199-206.

18. Choi G, Lee SH, Lokhande P, Kong BJ, Shim CS, et al. (2008) Percutaneous endoscopic approach for highly migrated intracanal disc herniations by foraminoplastic technique using rigid working channel endoscope. Spine 33: 508-515

19. Kim HS, Ju CI, Kim SW, Kim JG (2009) Endoscopic transforaminal suprapedicular approach in high grade inferior migrated lumbar disc herniation. J Kor Neurosurg Soc 45: 67-73.

20. Wu X, Fan G, Gu X, Guan X, He S (2016) Surgical outcome of two-level transforaminal percutaneous endoscopic lumbar discectomy for farmigrated disc herniation. BioMed Research International 2016: 11.

21. Ahn Y, Jang IT, Kim WK (2016) Transforaminal percutaneous endoscopic lumbar discectomy for very high-grade migrated disc herniation. Clin Neurol Neurosurg 147: 11-17.

22. Ahn Y (2012) Transforaminal percutaneous endoscopic lumbar discectomy: technical tips to prevent complications. Expert Review of Medical Devices 9: 361-366.

23. Guan X, Wu X, Fan G, Zhao S, Gu G, et al. (2016) Endoscopic retrieval of a broken guidewire during spinal surgery. Pain Physician 19: E339.
24. Choi KB, Lee CD, Lee SH (2010) Pyogenic spondylodiscitis after percutaneous endoscopic lumbar discectomy. J Korean Neurosurg Soc 48: 455-460.

25. Ahn Y, Lee SH (2012) Postoperative spondylodiscitis following transforaminal percutaneous endoscopic lumbar discectomy: Clinical characteristics and preventive strategies. Brit J Neurosurg 26: 482.

26. Lee SH, Kang BU, Ahn Y, Choi G, Choi YG, et al. (2006) Operative failure of percutaneous endoscopic lumbar discectomy: A radiologic analysis of 55 cases. Spine 31: E285-290.

27. Hsu HT, Chang SJ, Yang SS, Chai CL (2013) Learning curve of fullendoscopic lumbar discectomy. Eur Spine J 22: 727-733.

28. Lee DY, Lee SH (2008) Learning curve for percutaneous endoscopic lumbar discectomy. Neurol Med Chir (Tokyo) 48: 383-388.

29. Morgenstern R, Morgenstern C, Yeung AT (2007) The learning curve in foraminal endoscopic discectomy: Experience needed to achieve a $90 \%$ success rate. SAS J 1: 100-107.

30. Ahn SS, Kim SH, Kim DW (2015) Learning curve of percutaneous endoscopic lumbar discectomy based on the period (early vs. late) and technique (in-and-out vs. in-and-out-and-in): A retrospective comparative study. J Korean Neurosurg Soc 58: 539.

31. Wang H, Zhou Y, Li C, Liu J, Xiang L (2015) Risk factors for failure of single-level percutaneous endoscopic lumbar discectomy. J Neurosurg Spine 23: 320-325.

32. Kim HS, Park JY (2013) Comparative assessment of different percutaneous endoscopic interlaminar lumbar discectomy (PEID) techniques. Pain Physician 16: 359. 